PROCEEDINGS OF THE

AMERICAN MATHEMATICAL SOCIETY

Volume 137, Number 10, October 2009, Pages 3387-3397

S 0002-9939(09)09935-3

Article electronically published on May 6, 2009

\title{
THE ŁOJASIEWICZ EXPONENT OF AN ISOLATED WEIGHTED HOMOGENEOUS SURFACE SINGULARITY
}

\author{
TADEUSZ KRASIŃSKI, GRZEGORZ OLEKSIK, AND ARKADIUSZ PŁOSKI
}

(Communicated by Mei-Chi Shaw)

\begin{abstract}
We give an explicit formula for the Eojasiewicz exponent of an isolated weighted homogeneous surface singularity in terms of its weights. From the formula we get that the Lojasiewicz exponent is a topological invariant of these singularities.
\end{abstract}

\section{INTRODUCTION}

Let $f=f\left(z_{1}, \ldots, z_{n}\right) \in \mathbb{C}\left\{z_{1}, \ldots, z_{n}\right\}$ be a convergent power series defining an isolated singularity at the origin $\mathbf{0} \in \mathbb{C}^{n}$; i.e. $f(\mathbf{0})=0$ and the gradient of $f$,

$$
\nabla f:=\left(\frac{\partial f}{\partial z_{1}}, \ldots, \frac{\partial f}{\partial z_{n}}\right):\left(\mathbb{C}^{n}, \mathbf{0}\right) \rightarrow\left(\mathbb{C}^{n}, \mathbf{0}\right),
$$

has an isolated zero at $\mathbf{0} \in \mathbb{C}^{n}$. The Eojasiewicz exponent $\mathcal{L}_{0}(f)$ of $f$ is by definition the smallest $\theta>0$ such that there exists a neighbourhood $U$ of $\mathbf{0} \in \mathbb{C}^{n}$ and a constant $c>0$ such that

$$
|\nabla f(z)| \geqslant c|z|^{\theta} \text { for all } z \in U
$$

B. Teissier proved that $\mathcal{L}_{0}(f)+1$ is equal to the maximal polar invariant of the singularity $f([\mathrm{~T}]$, Corollary 2$)$. In particular $\mathcal{L}_{0}(f)$ depends only on the analytical type of the germ $\{f=0\}$ (even more: $\mathcal{L}_{0}(f)$ is an invariant of the "c-cosécance" introduced in [T] ). It is an open question whether $\mathcal{L}_{0}(f)$ is a topological invariant of an isolated singularity $f$. Let $\operatorname{Suff}_{0}(f)$ be the $C^{0}$-degree of sufficency of $f$, i.e. the smallest integer $r$ such that $f$ is topologically equivalent to $f+g$ for all $g$ with ord $g \geq r+1$. Then $\operatorname{Suff}_{0}(f)=\left[\mathcal{L}_{0}(f)\right]+1([\mathrm{~T}]$, Theorem 8), where $[a]$ is the integral part of $a \in \mathbb{R}$. The Łojasiewicz exponent can be calculated by means of analytic paths $\varphi(t)=\left(\varphi_{1}(t), \ldots, \varphi_{n}(t)\right) \in \mathbb{C}\{t\}^{n}, \varphi(0)=\mathbf{0}, \varphi(t) \neq 0$ in $\mathbb{C}\{t\}^{n}$. If $\operatorname{ord} \varphi:=\inf _{i=1}^{n} \operatorname{ord} \varphi_{i}$, then

$$
\mathcal{L}_{0}(f)=\sup _{\varphi} \frac{\operatorname{ord}((\nabla f) \circ \varphi)}{\operatorname{ord} \varphi}
$$

(by the Curve Selection Lemma; see also [L-JT]). In the two-dimensional case there are many explicit formulas for $\mathcal{L}_{0}(f)$ in various terms (see [KL, CK1], CK2, [L]). In this paper we investigate the problem of determining the Łojasiewicz exponent

Received by the editors July 10, 2008, and, in revised form, February 10, 2009.

2000 Mathematics Subject Classification. Primary 32S05.

Key words and phrases. Łojasiewicz exponent, weighted homogeneous polynomial, isolated surface singularity, polar curve. 
for weighted homogeneous isolated singularities. Let us recall that if $\left(w_{1}, \ldots, w_{n}\right)$ is a sequence of $n$ rational numbers (weights) such that $w_{i} \geq 2$ for $i=1, \ldots, n$, then a polynomial $f \in \mathbb{C}\left[z_{1}, \ldots, z_{n}\right]$ is called weighted homogeneous of type $\left(w_{1}, \ldots, w_{n}\right)$ if $f$ may be written as a sum of monomials $z_{1}^{\alpha_{1}} \ldots z_{n}^{\alpha_{n}}$ with

$$
\frac{\alpha_{1}}{w_{1}}+\ldots+\frac{\alpha_{n}}{w_{n}}=1 .
$$

For another definition of weighted homogeneous polynomials see the Appendix.

The set of weights $\left\{w_{1}, \ldots, w_{n}\right\}$ of a weighted homogeneous polynomial $f$ defining an isolated singularity is an analytic invariant of the germ $\{f=0\}[\underline{\mathbf{S}}$. Many topological invariants of weighted homogeneous isolated singularities are expressed in terms of weights: for instance, the Milnor number $\mu_{0}(f)$ of $f$ and the characteristic monodromy polynomial $\Delta_{f}(t)[\mathrm{MO}$, and in the case of weighted homogeneous isolated surface singularities, the multiplicity of $f[\mathbf{Y}]$, the fundamental group $\pi\left(K_{f}\right)$ of the link of $f$ and the minimal resolution of $f$ OW].

In this note we will give a formula for the Lojasiewicz exponent of weighted homogeneous isolated surface singularities in terms of its weights. Precisely, the Łojasiewicz exponent is equal to the maximum of its weights minus one. As a corollary we obtain that in this class of singularities $\mathcal{L}_{0}(f)$ is a topological invariant.

Estimations of the Lojasiewicz exponent for quasi-homogeneous isolated singularities in the real and complex cases are in a recent preprint by Haraux and Pham $[\mathrm{HP}$. Estimations in the general case can be found in [Lt], [F], [P1], $\mathrm{A}$.

\section{RESUlts}

The main result of this paper is the following:

Theorem 1. Let $f=f\left(z_{1}, z_{2}, z_{3}\right)$ be a weighted homogeneous polynomial of type $\left(w_{1}, w_{2}, w_{3}\right)$ defining an isolated singularity at the origin $\mathbf{0} \in \mathbb{C}^{3}$. Then

$$
\mathcal{L}_{0}(f)=\max _{i=1}^{3}\left(w_{i}-1\right) .
$$

An analogous formula also holds in the case $n=2$ (Corollary 4). In the general case we have only the inequality " $\leq$ " in (2.1); the equality holds under additional assumptions (Propositions 1 and 2 in Section 3).

The proof of the above theorem is given in Section 5 .

Corollary 1. $\operatorname{Suff}_{0}(f)=\left[\max _{i=1}^{3}\left(w_{i}\right)\right]$.

Since weights are a topological invariant of weighted homogeneous surface singularities [Y], Theorem B, we obtain

Corollary 2. The Eojasiewicz exponent $\mathcal{L}_{0}(f)$ of weighted homogeneous isolated surface singularities $f$ is a topological invariant.

It means that if $f, f^{\prime}$ are weighted homogeneous isolated surface singularities and $\left(\mathbb{C}^{3}, V(f), \mathbf{0}\right)$ is homeomorphic to $\left(\mathbb{C}^{3}, V\left(f^{\prime}\right), \mathbf{0}\right)$, then $\mathcal{L}_{0}(f)=\mathcal{L}_{0}\left(f^{\prime}\right)$.

From Corollary 1 we easily get

Corollary 3. $\operatorname{deg} f \leq \operatorname{Suff}_{0}(f)$.

The above inequality may be strict. 
Example 1. Let $a, b$ be integers such that $b \geq 2$ and $\frac{a}{2}>b-1$. The polynomial $f=z_{1}^{a} z_{2}+z_{2}^{b}+z_{3}^{2}$ is of type $\left(\frac{a b}{b-1}, b, 2\right)$ and defines an isolated singularity at $\mathbf{0} \in \mathbb{C}^{3}$. Then $\operatorname{deg} f=a+1$ and $\operatorname{Suff}_{0}(f)=\left[\frac{a b}{b-1}\right]>\operatorname{deg} f$.

The crucial role in the proof of the main theorem is played by the following result concerning arbitrary isolated surface singularities.

Theorem 2. Let $f=f\left(z_{1}, z_{2}, z_{3}\right)$ be an isolated surface singularity and

$$
V\left(\frac{\partial f}{\partial z_{2}}, \frac{\partial f}{\partial z_{3}}\right) \subset V\left(z_{1}\right) .
$$

Then

$$
z_{1} \in\left(\frac{\partial f}{\partial z_{2}}, \frac{\partial f}{\partial z_{3}}\right) \quad \text { in } \mathbb{C}\left\{z_{1}, z_{2}, z_{3}\right\} .
$$

The proof of the above theorem is given in Section 4.

To generalize Theorem 1 to the $n$-dimensional case it is enough to prove the last theorem in the $n$-dimensional case in the following formulation.

Problem 1. Let $f=f\left(z_{1}, \ldots, z_{n}\right)$ be an isolated singularity and

$$
V\left(\frac{\partial f}{\partial z_{2}}, \ldots, \frac{\partial f}{\partial z_{n}}\right) \subset V\left(z_{1}\right) .
$$

Then

$$
z_{1} \in\left(\frac{\partial f}{\partial z_{2}}, \ldots, \frac{\partial f}{\partial z_{n}}\right) \text { in } \mathbb{C}\left\{z_{1}, \ldots, z_{n}\right\} .
$$

Remark 1. Theorem 10 implies that the maximal polar invariant of a weighted homogeneous isolated surface singularity is equal to its maximal weight.

\section{UPPER BOUND FOR THE ŁOJASIEWICZ EXPONENT OF WEIGHTED HOMOGENEOUS ISOLATED SINGULARITIES}

In this section we will prove

Proposition 1. Let $f \in \mathbb{C}\left\{z_{1}, \ldots, z_{n}\right\}$ be a weighted homogeneous isolated singularity of type $\left(w_{1}, \ldots, w_{n}\right)$ at $\mathbf{0} \in \mathbb{C}^{n}$. Then

$$
\mathcal{L}_{0}(f) \leq \max _{i=1}^{n}\left(w_{i}-1\right) .
$$

Remark 2. If $f$ is a homogeneous isolated singularity of degree $d>1$, then $\mathcal{L}_{0}(f)=$ $d-1$ ([2], Lemma 2.4). In this case we have $w_{i}=d$ for $i=1, \ldots, n$.

We will get Proposition 1 from an estimation of the Lojasiewicz exponent for semi-weighted homogeneous mappings given in [P2] (see also [F], Theorem 3.2). First we recall the notion of the Łojasiewicz exponent for holomorphic mappings with an isolated zero.

Let $\boldsymbol{f}=\left(f_{1}, \ldots, f_{n}\right) \in \mathbb{C}\left\{z_{1}, \ldots, z_{n}\right\}^{n}$ define a germ of the holomorphic mapping $\boldsymbol{f}:\left(\mathbb{C}^{n}, \mathbf{0}\right) \rightarrow\left(\mathbb{C}^{n}, \mathbf{0}\right)$ with an isolated zero at $\mathbf{0} \in \mathbb{C}^{n}$. The Lojasiewicz exponent $l_{0}(\boldsymbol{f})$ of $\boldsymbol{f}$ is by definition the smallest $\theta>0$ such that there exist a neighbourhood $U$ of $\mathbf{0} \in \mathbb{C}^{n}$ and a constant $c>0$ such that

$$
|\boldsymbol{f}(z)| \geqslant c|z|^{\theta} \text { for all } z \in U .
$$

Clearly $\mathcal{L}_{0}(f)=l_{0}(\nabla f)$. 
Lemma 1. Let $f_{i}$ for $i=1, \ldots, n$ be a polynomial whose support $\operatorname{supp} f_{i}$ lies in the hyperplane $q_{1} \alpha_{1}+\ldots+q_{n} \alpha_{n}=d_{i}$, where $q_{1}, \ldots, q_{n}, d_{i}>0$ are integers. Suppose that $\boldsymbol{f}=\left(f_{1}, \ldots, f_{n}\right)$ has an isolated zero at $\mathbf{0} \in \mathbb{C}^{n}$. Then

$$
l_{0}(\boldsymbol{f}) \leq \frac{\max _{i=1}^{n}\left(d_{i}\right)}{\min _{i=1}^{n}\left(q_{i}\right)} .
$$

Proof. See [P2], Proposition 2.2.

Now we can give

Proof of Proposition 1. Let $q_{1}, \ldots, q_{n}$ and $d$ be positive integers such that $q_{i} w_{i}=d$ for $i=1, \ldots, n$. Since $f$ is an isolated singularity we have $\frac{\partial f}{\partial z_{i}} \neq 0$ for $i=1, \ldots, n$. Obviously $\operatorname{supp}\left(\frac{\partial f}{\partial z_{i}}\right)$ lies on the hyperplane $q_{1} \alpha_{1}+\ldots+q_{n} \alpha_{n}=d-q_{i}$. Using Lemma 1 we get

$$
\mathcal{L}_{0}(f)=l_{0}(\nabla f) \leq \frac{\max _{i=1}^{n}\left(d-q_{i}\right)}{\min _{i=1}^{n}\left(q_{i}\right)}=\max _{i=1}^{n}\left(\frac{d}{q_{i}}-1\right)=\max _{i=1}^{n}\left(w_{i}-1\right) .
$$

Let $f \in \mathbb{C}\left\{z_{1}, \ldots, z_{n}\right\}$ be an isolated singularity and let $l=\sum_{i=1}^{n} a_{i} z_{i}$ be a linear nonzero form. A (local) polar curve of $f$ related to $l$ is the germ $\Gamma_{l}(f)$ of the analytic set given by the equations

$$
\frac{\partial(f, l)}{\partial\left(z_{i}, z_{j}\right)}=0, \quad 1 \leq i<j \leq n
$$

near the origin. It is easy to check that $\operatorname{dim} \Gamma_{l}(f)=1$. In particular $\Gamma_{z_{k}}(f)$ is given by the equations

$$
\frac{\partial f}{\partial z_{1}}=\cdots=\frac{\partial f}{\partial z_{k-1}}=\frac{\partial f}{\partial z_{k+1}}=\cdots=\frac{\partial f}{\partial z_{n}}=0 .
$$

Proposition 2. Let $f \in \mathbb{C}\left\{z_{1}, \ldots, z_{n}\right\}$ be a weighted homogeneous isolated singularity of type $\left(w_{1}, \ldots, w_{n}\right)$. Suppose that $w_{k}=\max _{i=1}^{n}\left(w_{i}\right)$ and $\Gamma_{z_{k}}(f) \not \subset V\left(z_{k}\right)$. Then

$$
\mathcal{L}_{0}(f)=\max _{i=1}^{n}\left(w_{i}-1\right) .
$$

Proof. By Proposition 1 we have

$$
\mathcal{L}_{0}(f) \leq w_{k}-1 .
$$

To check that

$$
\mathcal{L}_{0}(f) \geq w_{k}-1
$$

we choose an open neighbourhood $U$ of $\mathbf{0} \in \mathbb{C}^{n}$ such that if $\nabla f(\boldsymbol{z})=0, \boldsymbol{z} \in U$, then $\boldsymbol{z}=\mathbf{0}$. From the assumption $\Gamma_{z_{k}}(f) \not \subset V\left(z_{k}\right)$ it follows that the system of equations (3.1) has in $U$ a solution $\boldsymbol{a}=\left(a_{1}, \ldots, a_{n}\right)$ such that $a_{k} \neq 0$. Let $q_{1}, \ldots, q_{n}$ and $d$ be integers such that $q_{i} w_{i}=d$ for $i=1, \ldots, n$. Set

$$
\boldsymbol{\varphi}(t)=\left(a_{1} t^{q_{1}}, \ldots, a_{n} t^{q_{n}}\right) .
$$


Since supp $\left(\frac{\partial f}{\partial z_{i}}\right)$ lies on the hyperplane $q_{1} \alpha_{1}+\ldots+q_{n} \alpha_{n}=d-q_{i}$ we get

$$
\begin{aligned}
& \frac{\partial f}{\partial z_{i}}(\boldsymbol{\varphi}(t))=t^{d-q_{i}} \frac{\partial f}{\partial z_{i}}(\boldsymbol{a})=0 \text { for } i \neq k, \\
& \frac{\partial f}{\partial z_{k}}(\boldsymbol{\varphi}(t))=t^{d-q_{k}} \frac{\partial f}{\partial z_{k}}(\boldsymbol{a}) \neq 0 .
\end{aligned}
$$

Therefore we get

$$
\mathcal{L}_{0}(f) \geq \frac{\operatorname{ord}((\nabla f) \circ \varphi(t))}{\operatorname{ord} \varphi(t)}=\frac{\operatorname{ord}\left(\frac{\partial f}{\partial z_{k}}(\boldsymbol{\varphi}(t))\right)}{\operatorname{ord} \boldsymbol{\varphi}(t)}=\frac{d-q_{k}}{q_{k}}=w_{k}-1 .
$$

The above propositions give the formula for the Eojasiewicz exponent in a simpler two-dimensional case.

Corollary 4. Let $f \in \mathbb{C}\left\{z_{1}, z_{2}\right\}$ be a weighted homogeneous isolated singularity of type $\left(w_{1}, w_{2}\right)$ at $\mathbf{0} \in \mathbb{C}^{2}$. Then

$$
\mathcal{L}_{0}(f)=\max _{i=1}^{2}\left(w_{i}-1\right)
$$

Proof. Assume that $w_{1} \leq w_{2}$. If $V\left(\frac{\partial f}{\partial z_{1}}\right) \not \subset V\left(z_{2}\right)$, then the corollary follows from Proposition 2. If $V\left(\frac{\partial f}{\partial z_{1}}\right) \subset V\left(z_{2}\right)$, then $z_{2}=A \frac{\partial f}{\partial z_{1}}$ in $\mathbb{C}\left\{z_{1}, z_{2}\right\}$. In fact, by the local Hilbert Nullstellensatz $z_{2}^{p}=A \frac{\partial f}{\partial z_{1}}$ in $\mathbb{C}\left\{z_{1}, z_{2}\right\}$ for some positive integer $p$. Assume that $p$ is the smallest possible. Then $z_{2}$ does not divide $A$. Since $\mathbb{C}\left\{z_{1}, z_{2}\right\}$ is a unique factorization domain we get $\frac{\partial f}{\partial z_{1}}=z_{2}^{p} B, B(0,0) \neq 0$. Hence there exist $C \in \mathbb{C}\left\{z_{1}, z_{2}\right\}$ and $g \in \mathbb{C}\left\{z_{2}\right\}, g(0)=0$, such that

$$
f\left(z_{1}, z_{2}\right)=z_{2}^{p} C\left(z_{1}, z_{2}\right)+g\left(z_{2}\right) \text { in } \mathbb{C}\left\{z_{1}, z_{2}\right\} .
$$

If we had $p>1$, then by condition $\frac{\partial f}{\partial z_{2}}(0,0)=0$ we would obtain $g^{\prime}(0)=0$. This would imply

$$
\frac{\partial f}{\partial z_{1}}\left(z_{1}, 0\right)=0 \text { and } \frac{\partial f}{\partial z_{2}}\left(z_{1}, 0\right)=0
$$

which contradicts the assumption that $f$ is an isolated singularity. So $p=1$, i.e. $z_{2}=A \frac{\partial f}{\partial z_{1}}$ in $\mathbb{C}\left\{z_{1}, z_{2}\right\}$. Hence $\frac{\partial^{2} f}{\partial z_{1} \partial z_{2}}(0,0) \neq 0$. This implies that the monomial $c z_{1} z_{2}$ appears with a nonzero coefficient $c \neq 0$ in the Taylor expansion of $f$. We then get $\frac{1}{w_{1}}+\frac{1}{w_{2}}=1$, which implies $w_{1}=w_{2}=2$ (by definition of weighted homogeneous polynomials $w_{1}, w_{2} \geq 2$ ). Thus $f$ is a homogeneous form of degree 2 and $\mathcal{L}_{0}(f)=1=\max _{i=1}^{2}\left(w_{i}-1\right)$ by Remark 2

Remark 3. It is well known that if $f=f\left(z_{1}, z_{2}\right)$ defines an isolated curve singularity, then the Milnor number $\mu_{0}(f)$ and the Lojasiewicz exponent $\mathcal{L}_{0}(f)$ are topological invariants of the germ $\{f=0\}$ ([T] $)$. Moreover, if additionally $f$ is weighted homogeneous of type $\left(w_{1}, w_{2}\right)$, then by [MO]

$$
\mu_{0}(f)=\left(w_{1}-1\right)\left(w_{2}-1\right),
$$

and by Corollary 4

$$
\mathcal{L}_{0}(f)=\max \left(\left(w_{1}-1\right),\left(w_{2}-1\right)\right)
$$


Hence the set of weights

$$
\left\{w_{1}, w_{2}\right\}=\left\{\frac{\mu_{0}(f)}{\mathcal{L}_{0}(f)}+1, \mathcal{L}_{0}(f)+1\right\}
$$

is also a topological invariant of the germ $\{f=0\}$.

\section{Proof of Theorem 2}

Proof. In the sequel we will use the following notation for any $P \in \mathbb{C}\left\{z_{1}, z_{2}, z_{3}\right\}$. Let $P=P_{0}+P_{1} z_{1}+P_{2} z_{1}^{2}+\ldots$ with $P_{i} \in \mathbb{C}\left\{z_{2}, z_{3}\right\}$ for $i=0,1,2, \ldots$ Then we put $\widehat{P}=P_{1}+P_{2} z_{1}+\ldots$ Thus $P_{0}=P\left(0, z_{2}, z_{3}\right)$ and $P=P_{0}+z_{1} \widehat{P}$ in $\mathbb{C}\left\{z_{1}, z_{2}, z_{3}\right\}$. Note that

$$
\left(\frac{\partial P}{\partial z_{2}}\right)_{0}=\frac{\partial P_{0}}{\partial z_{2}} \text { and }\left(\frac{\partial P}{\partial z_{3}}\right)_{0}=\frac{\partial P_{0}}{\partial z_{3}} \text {. }
$$

Let us pass to the proof of the theorem. We have to show that there exists a power series $A, B \in \mathbb{C}\left\{z_{1}, z_{2}, z_{3}\right\}$ such that

$$
z_{1}=A \frac{\partial f}{\partial z_{2}}+B \frac{\partial f}{\partial z_{3}} \text { in } \mathbb{C}\left\{z_{1}, z_{2}, z_{3}\right\} .
$$

It is easy to check the following three properties:

(1) The system of equations

$$
\frac{\partial f_{0}}{\partial z_{2}}=\frac{\partial f_{0}}{\partial z_{3}}=f_{1}=0
$$

has an isolated solution $z_{2}=z_{3}=0$ near the origin $\mathbf{0} \in \mathbb{C}^{2}$ (otherwise, $f$ does not define an isolated singularity).

(2) The analytic set defined by equations

$$
\frac{\partial f_{0}}{\partial z_{2}}=\frac{\partial f_{0}}{\partial z_{3}}=0
$$

near the origin $\mathbf{0} \in \mathbb{C}^{2}$ is of pure dimension one (since $\Gamma_{z_{1}}(f)$ is of pure dimension one and lies in $\left.\left\{z_{1}=0\right\}\right)$.

(3) For some integer $p>0$

$$
z_{1}^{p}=A \frac{\partial f}{\partial z_{2}}+B \frac{\partial f}{\partial z_{3}} \text { in } \mathbb{C}\left\{z_{1}, z_{2}, z_{3}\right\}
$$

(by the local Hilbert Nullstellensatz).

Assume that $p>0$ in (3) is the smallest possible. Hence $A_{0} \neq 0$ or $B_{0} \neq 0$. Then we have the following fact.

Property 1. $A_{0} \not \equiv 0\left(\bmod \frac{\partial f_{0}}{\partial z_{3}}\right)$ or $B_{0} \not \equiv 0\left(\bmod \frac{\partial f_{0}}{\partial z_{2}}\right)$ in $\mathbb{C}\left\{z_{2}, z_{3}\right\}$.

Proof of Property 1. Suppose that $A_{0} \equiv 0\left(\bmod \frac{\partial f_{0}}{\partial z_{3}}\right)$; that is, $A_{0}=\widetilde{A_{0}} \frac{\partial f_{0}}{\partial z_{3}}$ in $\mathbb{C}\left\{z_{2}, z_{3}\right\}$. Then

$$
\begin{aligned}
A & =A_{0}+z_{1} \widehat{A}=\widetilde{A_{0}} \frac{\partial f_{0}}{\partial z_{3}}+z_{1} \widehat{A}=\widetilde{A_{0}}\left(\frac{\partial f}{\partial z_{3}}-z_{1} \frac{\partial \widehat{f}}{\partial z_{3}}\right)+z_{1} \widehat{A} \\
& =\widetilde{A_{0}} \frac{\partial f}{\partial z_{3}}+z_{1} C \text { in } \mathbb{C}\left\{z_{1}, z_{2}, z_{3}\right\} .
\end{aligned}
$$


From (3) we get

$$
\begin{aligned}
z_{1}^{p} & =\left(\widetilde{A_{0}} \frac{\partial f}{\partial z_{3}}+z_{1} C\right) \frac{\partial f}{\partial z_{2}}+B \frac{\partial f}{\partial z_{3}} \\
& =\left(\widetilde{A_{0}} \frac{\partial f}{\partial z_{2}}+B\right) \frac{\partial f}{\partial z_{3}}+z_{1} C \frac{\partial f}{\partial z_{2}}
\end{aligned}
$$

By minimality of $p$ we get $\widetilde{A_{0}} \frac{\partial f}{\partial z_{2}}+B \not \equiv 0\left(\bmod z_{1}\right)$, and consequently $\frac{\partial f}{\partial z_{3}} \equiv 0$ $\left(\bmod z_{1}\right)$, which implies $\frac{\partial f_{0}}{\partial z_{3}}=0$ in $\mathbb{C}\left\{z_{2}, z_{3}\right\}$. Similarly the condition $B_{0} \equiv 0$ $\left(\bmod \frac{\partial f_{0}}{\partial z_{2}}\right)$ implies $\frac{\partial f_{0}}{\partial z_{2}}=0$ in $\mathbb{C}\left\{z_{2}, z_{3}\right\}$. This proves Property 1 .

From (3) we get

$$
A_{0} \frac{\partial f_{0}}{\partial z_{2}}+B_{0} \frac{\partial f_{0}}{\partial z_{3}}=0 \text { in } \mathbb{C}\left\{z_{2}, z_{3}\right\} .
$$

Suppose to the contrary that $p>1$. Then differentiating the equality in (3) and putting $z_{1}=0$ we get

$$
A_{0} \frac{\partial f_{1}}{\partial z_{2}}+B_{0} \frac{\partial f_{1}}{\partial z_{3}}+A_{1} \frac{\partial f_{0}}{\partial z_{2}}+B_{1} \frac{\partial f_{0}}{\partial z_{3}}=0 \text { in } \mathbb{C}\left\{z_{2}, z_{3}\right\} .
$$

From (2) it follows that we may write

$$
f_{0}=g_{0} g_{1}^{k_{1}} \ldots g_{r}^{k_{r}} \quad \text { in } \mathbb{C}\left\{z_{2}, z_{3}\right\}
$$

where $k_{i} \geq 2$ for $i=1, \ldots, r, r \geq 1, g_{i}$ are irreducible and $g_{i}$ does not divide $g_{j}$ in $\mathbb{C}\left\{z_{2}, z_{3}\right\}$ for $i \neq j$. Note that

$$
\operatorname{GCD}\left(\frac{\partial f_{0}}{\partial z_{2}}, \frac{\partial f_{0}}{\partial z_{3}}\right)=g_{1}^{k_{1}-1} \ldots g_{r}^{k_{r}-1}
$$

Property 2. There exists an $i \in\{1, \ldots, r\}$ such that

$$
\frac{\partial\left(g_{i}, f_{1}\right)}{\partial\left(z_{2}, z_{3}\right)} \equiv 0\left(\bmod g_{i}\right) \text {. }
$$

Proof of Property 2. Using Properties (4.1), (4.3) and Property 1 we check that

$$
A_{0} \not \equiv 0\left(\bmod \operatorname{GCD}\left(\frac{\partial f_{0}}{\partial z_{2}}, \frac{\partial f_{0}}{\partial z_{3}}\right)\right)
$$

or

$$
B_{0} \not \equiv 0\left(\bmod \operatorname{GCD}\left(\frac{\partial f_{0}}{\partial z_{2}}, \frac{\partial f_{0}}{\partial z_{3}}\right)\right) .
$$

Therefore there is an $i \in\{1, \ldots, r\}$ such that

$$
A_{0} \not \equiv 0\left(\bmod g_{i}^{k_{i}-1}\right) \quad \text { or } \quad B_{0} \not \equiv 0\left(\bmod g_{i}^{k_{i}-1}\right) \text {. }
$$

We may suppose $i=1$. Write $f_{0}=g_{1}^{k_{1}} \widehat{g_{1}}$ in $\mathbb{C}\left\{z_{2}, z_{3}\right\}$. Obviously $\widehat{g_{1}} \not \equiv 0\left(\bmod g_{1}\right)$. Using (4.1) after a simple calculation we get

$$
A_{0}\left(k_{1} \frac{\partial g_{1}}{\partial z_{2}} \widehat{g_{1}}+g_{1} \frac{\partial \widehat{g_{1}}}{\partial z_{2}}\right)+B_{0}\left(k_{1} \frac{\partial g_{1}}{\partial z_{3}} \widehat{g_{1}}+g_{1} \frac{\partial \widehat{g_{1}}}{\partial z_{3}}\right)=0 \text { in } \mathbb{C}\left\{z_{2}, z_{3}\right\} .
$$

Hence for each integer $m \geq 0$

$$
A_{0} \equiv 0\left(\bmod g_{1}^{m}\right) \text { if and only if } B_{0} \equiv 0\left(\bmod g_{1}^{m}\right) \text {. }
$$


Therefore we can write $A_{0}=A_{0}^{\prime} g_{1}^{m_{1}}$ and $B_{0}=B_{0}^{\prime} g_{1}^{m_{1}}$, where $0 \leq m_{1}<k_{1}-1$ and $A_{0}^{\prime} \not \equiv 0\left(\bmod g_{1}\right), B_{0}^{\prime} \not \equiv 0\left(\bmod g_{1}\right)$. From (4.1) and (4.2) we get

$$
A_{0}^{\prime} \frac{\partial g_{1}}{\partial z_{2}}+B_{0}^{\prime} \frac{\partial g_{1}}{\partial z_{3}} \equiv 0\left(\bmod g_{1}\right)
$$

and

$$
A_{0}^{\prime} \frac{\partial f_{1}}{\partial z_{2}}+B_{0}^{\prime} \frac{\partial f_{1}}{\partial z_{3}} \equiv 0\left(\bmod g_{1}\right)
$$

Using Cramer's rule to (4.5) and (4.6) we get

$$
A_{0}^{\prime} \frac{\partial\left(g_{1}, f_{1}\right)}{\partial\left(z_{2}, z_{3}\right)} \equiv 0\left(\bmod g_{1}\right)
$$

and Property 2 follows since $A_{0}^{\prime} \not \equiv 0\left(\bmod g_{1}\right)$ and $g_{1}$ is irreducible.

We omit the simple proof of the next property.

Property 3. Let $P, Q \in \mathbb{C}\{x, y\}$ be power series in two variables $x, y$ without constant term. Let $P$ be irreducible and let $\frac{\partial(P, Q)}{\partial(x, y)} \equiv 0(\bmod P)$. Then $Q \equiv 0$ $(\bmod P)$.

Now we can finish the proof of Theorem 2, The assumption $p>1$ implies by Properties 2 and 3 that $f_{1}$ vanishes on a branch $V\left(g_{i}\right)$ of the curve $V\left(\frac{\partial f_{0}}{\partial z_{2}}, \frac{\partial f_{0}}{\partial z_{3}}\right)$. This contradicts property (1). Therefore $p=1$, which ends the proof.

\section{Proof of Theorem 1}

Let $f=f\left(z_{1}, z_{2}, z_{3}\right)$ be a weighted homogeneous polynomial of type $\left(w_{1}, w_{2}, w_{3}\right)$ defining an isolated singularity at the origin $\mathbf{0} \in \mathbb{C}^{3}$. We may assume that $w_{1}=$ $\max \left(w_{1}, w_{2}, w_{3}\right)$. If $\Gamma_{z_{1}}(f) \not \subset V\left(z_{1}\right)$, then $\mathcal{L}_{0}(f)=w_{1}-1$ by Proposition 2 . Suppose then that $\Gamma_{z_{1}}(f) \subset V\left(z_{1}\right)$. By Theorem 2 there exists a power series $A, B \in$ $\mathbb{C}\left\{z_{1}, z_{2}, z_{3}\right\}$ such that $z_{1}=A \frac{\partial f}{\partial z_{2}}+B \frac{\partial f}{\partial z_{3}}$. Differentiating and putting $z_{1}=z_{2}=$ $z_{3}=0$ we obtain

$$
\frac{\partial^{2} f}{\partial z_{1} \partial z_{2}}(\mathbf{0}) \neq 0 \text { or } \frac{\partial^{2} f}{\partial z_{1} \partial z_{3}}(\mathbf{0}) \neq 0 .
$$

Thus the support $\operatorname{supp} f$ contains point $(1,1,0)$ or $(1,0,1)$. Hence $w_{1}=w_{2}=2$ or $w_{1}=w_{3}=2$. Since $w_{1}=\max \left(w_{1}, w_{2}, w_{3}\right)$, then $w_{1}=w_{2}=w_{3}=2$ and $f$ is homogeneous of degree 2 . Consequently $\mathcal{L}_{0}(f)=1=w_{1}-1$ by Remark 2 , and the theorem is proved.

Remark 4. Let $f=f_{0}+f_{1} z_{1}+f_{2} z_{1}^{2}+\ldots$ with $f_{i} \in \mathbb{C}\left\{z_{2}, z_{3}\right\}$ for $i=0,1, \ldots$ be an isolated surface singularity such that $\Gamma_{z_{1}}(f) \subset V\left(z_{1}\right)$. From the proofs of Theorems 1 and 2 it follows that $f_{0}$ has a multiple factor and ord $f_{1}=1$. In particular ord $f=2$.

\section{Appendix}

There is another (weaker) definition of a weighted homogeneous polynomial. A polynomial $f \in \mathbb{C}\left[z_{1}, \ldots, z_{n}\right]$ is called a weak weighted homogeneous polynomial if there exist $n$ rational positive numbers (weights) $\left(w_{1}, \ldots, w_{n}\right)$ such that $f$ may be written as a sum of monomials $z_{1}^{\alpha_{1}} \ldots z_{n}^{\alpha_{n}}$ with

$$
\frac{\alpha_{1}}{w_{1}}+\ldots+\frac{\alpha_{n}}{w_{n}}=1
$$


Observe that we don't assume here that $w_{i} \geq 2$ for $i=1, \ldots, n$. The weights are not uniquely determined by the weak weighted homogeneous polynomial. If a weak weighted homogeneous polynomial $f$ of type $\left(w_{1}, \ldots, w_{n}\right)$ defines an isolated singularity at the origin, then $w_{i}>1$ for all $i=1, \ldots, n$ and

$$
\mu_{0}(f)=\prod_{i=1}^{n}\left(w_{i}-1\right)
$$

([MO], Theorem 1). The class of weak weighted homogeneous polynomials is broader than the class of weighted homogeneous polynomials. However, we can extend our main theorem to this class.

Theorem 3. Let $f=f\left(z_{1}, z_{2}, z_{3}\right)$ be a weak weighted homogeneous polynomial of type $\left(w_{1}, w_{2}, w_{3}\right)$ defining an isolated singularity at the origin. Then

$$
\mathcal{L}_{0}(f)=\min \left(\max _{i=1}^{3}\left(w_{i}-1\right), \prod_{i=1}^{3}\left(w_{i}-1\right)\right) .
$$

Note that if $w_{i} \geq 2$ for all $i=1,2,3$, then $\max _{i=1}^{3}\left(w_{i}-1\right) \leq \prod_{i=1}^{3}\left(w_{i}-1\right)$ and we recover Theorem 1 .

In the proof we need the following useful lemma:

Lemma 2. Let $f \in \mathbb{C}\left\{z_{1}, \ldots, z_{n}\right\}$ define an isolated singularity at the origin. Then

$$
\mathcal{L}_{0}(f) \leq \mu_{0}(f)
$$

with equality if

$$
\operatorname{rk}\left(\frac{\partial^{2} f}{\partial z_{i} \partial z_{j}}(0)\right) \geq n-1
$$

Proof. It is well known that the monomials $z_{1}^{\mu}, \ldots, z_{n}^{\mu}, \mu=\mu_{0}(f)$, belong to the ideal $\left(\frac{\partial f}{\partial z_{1}}, \ldots, \frac{\partial f}{\partial z_{n}}\right)$. Whence the inequality $\mathcal{L}_{0}(f) \leq \mu_{0}(f)$ follows. If (6.1) holds, then we may assume, by the splitting lemma, that $f=z_{1}^{2}+\ldots+z_{n-1}^{2}+z_{n}^{\mu}$. This obviously implies $\mathcal{L}_{0}(f)=\mu_{0}(f)$.

Remark 5. One can prove that the equality $\mathcal{L}_{0}(f)=\mu_{0}(f)$ implies the inequality (6.1)

Proof of Theorem 3 . We get $\mathcal{L}_{0}(f) \leq \mu_{0}(f)=\prod_{i=1}^{3}\left(w_{i}-1\right)$ by the Milnor-Orlik formula. On the other hand our proof of Proposition 1 is valid in the case of weak weighted homogeneous isolated singularities, and consequently $\mathcal{L}_{0}(f) \leq$ $\max _{i=1}^{3}\left(w_{i}-1\right)$. Summing up we obtain the bound

$$
\mathcal{L}_{0}(f) \leq \min \left(\max _{i=1}^{3}\left(w_{i}-1\right), \prod_{i=1}^{3}\left(w_{i}-1\right)\right)
$$


To prove the opposite inequality we suppose, to the contrary, that we have strict " $<$ " inequality in (6.2). Then

$$
\begin{aligned}
& \mathcal{L}_{0}(f)<\max _{i=1}^{3}\left(w_{i}-1\right), \\
& \mathcal{L}_{0}(f)<\prod_{i=1}^{3}\left(w_{i}-1\right) .
\end{aligned}
$$

We may assume that $\max _{i=1}^{3}\left(w_{i}\right)=w_{1}$. Inequality (6.3) implies $V\left(\frac{\partial f}{\partial z_{2}}, \frac{\partial f}{\partial z_{3}}\right) \subset$ $V\left(z_{1}\right)$ (cf. the proof of Theorem 1). Using Remark 4 we check that, up to a permutation of variables $z_{2}, z_{3}$,

$$
f\left(z_{1}, z_{2}, z_{3}\right)=a z_{3}^{k}+b z_{1} z_{2}+z_{1}^{2} g\left(z_{1}, z_{3}\right),
$$

where $g\left(z_{1}, z_{3}\right)$ is a polynomial, $a b \neq 0$, and $k \geq 2$. Using Lemma 2 we check that $\mathcal{L}_{0}(f)=\mu_{0}(f)$. Since $\mu_{0}(f)=\prod_{i=1}^{3}\left(w_{i}-1\right)$ by the Milnor-Orlik formula, then $\mathcal{L}_{0}(f)=$ $\prod_{i=1}^{3}\left(w_{i}-1\right)$, which contradicts (6.4).

\section{REFERENCES}

[A] Abderrahmane, O. M.: On the Eojasiewicz exponent and Newton polyhedron. Kodai. Math. J. 28 (2005), 106-110. MR.2122194(2006c:32031)

[CK1] Chadzyński, J. and Krasiński, T.: The Eojasiewicz exponent of an analytic mapping of two complex variables at an isolated zero. In: Singularities, Banach Center Publ. 20, PWN, Warszawa, 1988, 139-146. MR.1101835 (92e:32018)

[CK2] Chadzyński, J. and Krasiński, T.: Resultant and the Eojasiewicz exponent. Ann. Polon. Math. 61 (1995), 95-100. MR.1318321 (96a:32061)

[F] Fukui, T.: Eojasiewicz type inequalities and Newton diagrams. Proc. Amer. Math. Soc. 112 (1991), 1169-1183. MR.1065945 (91j:58021)

[HP] Haraux, A. and Pham, T. S.: On the Eojasiewicz exponents of quasi-homogeneous functions. Preprints of the Laboratoire Jacques-Louis Lions 2007, Université Pierre et Marie Curie, No. R07041 (http://www.ann.jussieu.fr/publications/2007/R07041.pdf).

[KL] Kuo, T. C. and Lu, Y. C.: On analytic function germs of two complex variables. Topology 16 (1977), 299-310. MR0460711(57:704)

[L-JT] Lejeune-Jalabert, M. and Teissier, B.: Cloture integrale des idéaux et equisingularité. École Polytechnique, 1974.

[L] Lenarcik, A.: On the Eojasiewicz exponent of the gradient of a holomorphic function. In: Singularities Symposium-Łojasiewicz 70. Banach Center Publ. 44, PWN, Warszawa, 1998, 149-166. MR1677363 (2000h:32039)

[Lt] Lichtin, B.: Estimation of Łojasiewicz exponents and Newton polygons. Inventiones Math. 64 (1981), 417-429. MR632982 (83b:32006)

[MO] Milnor, J. and Orlik, P.: Isolated singularities defined by weighted homogeneous polynomials, Topology 9 (1970), 385-393. MR0293680 (45:2757)

[OW] Orlik, P. and Wagreich, P.: Isolated singularities of algebraic surfaces with $\mathbb{C}^{*}$-action. Ann. of Math. 93 (1971), 205-228. MR0284435 (44:1662)

[P1] Płoski, A.: Sur l'exposant d'une application analytique I. Bull. Polish Acad. Sci. Math. 32 (1984), 669-673. MR786190 (86j:32025a)

[P2] Płoski, A.: Sur l'exposant d'une application analytique II. Bull. Polish Acad. Sci. Math. 33 (1985), 123-127. MR805025 (86j:32025b)

[S] Saito, K.: Quasihomogene isolierte Singularitäten von Hyperflächen. Invent. Math. 14 (1971), 123-142. MR0294699 (45:3767) 
[T] Teissier, B.: Variétés polaires. Invent. Math. 40 (1977), 267-292. MR0470246 (57:10004)

[Y] Yau, S. S.-T.: Topological types and multiplicities of isolated quasihomogeneous surface singularities. Bull. Amer. Math. Soc. (N.S.) 19 (1988), 447-454. MR 935021 (92b:32042)

Faculty of Mathematics and Computer Science, University of Łódź, Banacha 22, 90-238 ŁóDź, POLAND

E-mail address: krasinsk@uni.lodz.pl

Faculty of Mathematics and Computer Science, University of Łódź, Banacha 22, 90-238 ŁÓDŹ, POLAND

E-mail address: oleksig@math.uni.lodz.pl

Department of Mathematics, Technical University, Al. 1000 LPP 7, 25-314 Kielce, POLAND

E-mail address: matap@tu.kielce.pl 